\title{
Dysregulation of TMPRSS3 and TNFRSF11B correlates with tumorigenesis and poor prognosis in patients with breast cancer
}

\author{
PING LUO $^{1 *}$, GAIXIA LU $^{2 *}$, LIN-LIN FAN ${ }^{3 *}$, XIAOMING ZHONG ${ }^{4,5^{*}}$, HUIQIONG YANG ${ }^{2}$, RUTING XIE ${ }^{2}$, \\ ZHONGWEI LV ${ }^{2}$, QIAN-ZHOU LV ${ }^{3}$, DA FU ${ }^{2}$, LI-XIN YANG ${ }^{6}$ and YUSHUI MA ${ }^{2,7}$ \\ ${ }^{1}$ Department of Breast Cancer, Nanchang Third Hospital, Nanchang, Jiangxi 330002; ${ }^{2}$ Department of Nuclear Medicine, \\ Shanghai 10th People's Hospital, Tongji University School of Medicine, Shanghai 200072; ${ }^{3}$ Department of Pharmacy, \\ Zhongshan Hospital, Fudan University, Shanghai 200032; ${ }^{4}$ Department of Radiology, Jiangxi Provincial Tumor Hospital, \\ Nanchang, Jiangxi 330029; ${ }^{5}$ Department of Radiology, Ganzhou City People's Hospital, Ganzhou, Jiangxi 341000; \\ ${ }^{6}$ Department of Thoracic Surgery, Changhai Hospital of Second Military Medical University, Shanghai 200433; \\ ${ }^{7}$ Shanghai Engineering Research Center of Molecular Therapeutics and New Drug Development, College of Chemistry \\ and Molecular Engineering, East China Normal University, Shanghai 200062, P.R. China
}

Received August 1, 2016; Accepted September 2, 2016

DOI: $10.3892 /$ or.2017.5449

\begin{abstract}
The present study was carried out to investigate the clinical significance of TMPRSS3 and TNFRSF11B in breast cancer. Thus, the expression levels of TMPRSS3 and TNFRSF11B and the correlation with prognosis in patients with breast cancer were analyzed in silico using gene microarray and hierarchical clustering analysis. Then, the differential expression in breast cancer vs. normal breast tissue was explored in the Oncomine platform and verified in our independent samples using IHC technique. Our results indicated that TMPRSS3 was upregulated and TNFRSF11B was downregulated in breast cancer tissues compared with the levels in the human normal breast tissues. TMPRSS3 and TNFRSF11B were confirmed to be correlated with distant organ metastasis of breast cancer. Moreover, upregulation of TMPRSS3 accompanied by downregulation of TNFRSF11B was found to be associated with a shorter median overall survival and indicated a poor prognosis. In conclusion, TMPRSS3 and TNFRSF11B may have potential prognostic value to be used as tumor biomarkers in breast cancer patients.
\end{abstract}

Correspondence to: Dr Da Fu or Dr Yushui Ma, Department of Nuclear Medicine, Shanghai 10th People's Hospital, Tongji University School of Medicine, Shanghai 200072, P.R. China

E-mail: fu800da900@126.com

E-mail: mayushui2015@126.com

${ }^{*}$ Contributed equally

Key words: breast cancer, TMPRSS3, TNFRSF11B, metastasis, prognosis

\section{Introduction}

Breast cancer is the second leading cause of cancer-related death among women after lung cancer, accounting for $23 \%$ of all cases of cancer in women (1). According to the American Cancer Society's estimates for 2016, 246,660 new cases of invasive breast cancer are expected to be diagnosed and $\sim 40,450$ women may die from breast cancer in the US (2). In China, the incidence and mortality of breast cancer have progressively increased during the past few decades (3). There are several treatment strategies including surgery, chemotherapy, radiation, hormone and targeted therapies, for breast cancer, which are dependent on various factors, such as the type of breast cancer, its stage and other special situations.

Breast cancer is a molecular heterogeneous malignancy, which can be divided into three basic types based on their immunohistochemical properties (receptor status): HR-positive, human epidermal growth factor receptor 2-positive (HER-2 ${ }^{+}$, and triple-negative breast cancers (4). The receptor status of a breast cancer is a vital factor in treatment decisions, since it determines whether the tumor can be treated with hormone or targeted therapy; this has significantly improved the prognosis of breast cancer patients $(5,6)$. Approximately $70 \%$ of breast cancers are ER $\alpha$-positive. ER $\alpha$-positivity is a crucial factor for deciding whether breast cancer patients can benefit from anti-estrogen therapy, which leads to a significant reduction in the mortality of breast cancer patients (7). However, despite advances in first-line endocrine therapy of advanced HR-positive breast cancer, cancer recurrence and subsequent drug resistance appear to be inevitable and present serious obstacles to successful treatment $(8,9)$. Therefore, it is essential to explore new molecular markers to identify the subgroup of breast tumors and to identify prognostic markers to improve the outcome of breast cancer patients. 
TMPRSS3 encodes a protein that belongs to the serine protease family, which participate in various biological processes, and whose dysregulation often leads to human diseases and disorders. TMPRSS3 has been reported as a tumor-associated gene that is overexpressed in pancreatic cancer (10) and ovarian tumors. It promotes the proliferation, invasion and migration of ovarian cancer cells (11). As an important tumor-associated gene, TMPRSS3 expression and its prognostic value in breast cancer has been reported, but the results are controversial $(12,13)$.

TNFRSF11B, also called osteoprotegerin (OPG) or osteoclastogenesis inhibitory factor, is a cytokine receptor belonging to the tumor necrosis factor (TNF) receptor superfamily. OPG is a decoy receptor for the receptor activator of nuclear factor- $\kappa \mathrm{B}$ ligand (RANKL), which blocks RANKL-RANK interaction by binding to RANKL (14). Receptor activator of nuclear factor- $\kappa \mathrm{B}$ and its ligand (RANK/RANKL) and OPG are key molecules for bone metabolism. The RANK/RANKL/ OPG pathway plays an important role in the development and progression of bone metastasis in various types of cancers (15). However, little is known concerning the role of OPG in breast cancer prognosis. Therefore, we aimed to evaluate OPG expression and the associated clinical significance in breast cancer.

Although, TMPRSS3 and TNFRSF11B have been found to play an important role in breast cancer, the expression levels and the clinical significance of TMPRSS3 and TNFRSF11B in breast cancer remain unclear. In the present study, we analyzed TMPRSS3 and TNFRSF11B expression and explored their prognostic value to provide useful insight into the development of more effective targeted therapies for breast cancer.

\section{Materials and methods}

Statement of ethics. The study protocol and acquisition of tissue specimens were approved by the Ethics Committee of Ganzhou City People's Hospital (2015-RES-15). Each participant provided written informed consent before participating in the present study.

Patients and tissue samples. The paraffin specimens used for immunohistochemistry were collected from 86 breast cancer patients undergoing surgical resection and were classified according to the most recent World Health Organization (WHO) classification which was confirmed by two experienced pathologists independently at the Shanghai 10th People's Hospital, Tongji University School of Medicine between 2007 and 2015. Clinical data were collected from patient operative and pathological records, and follow-up data were gathered by telephone or direct correspondence. The time of tumor relapse or death was verified by the patient or their relatives, by medical recording, or by the social security record. Overall survival (OS) was calculated in months from the date of diagnosis to the time of death, regardless of cause. Disease-free survival (DFS) was defined as the period from the initial date of diagnosis to the time of tumor progression, or to the time of death due to the disease.

Bioinformatic analysis. The expression levels of TMPRSS3 and TNFRSF11B were investigated in a gene microarray from the GEO database. Hierarchical clustering was performed using the multiple experiment viewer (MeV) 4.9.0 software (http://www.tm4.org/mev/).

Immunohistochemistry and $H \& E$ staining. Immunohistochemical and hematoxylin and eosin (H\&E) staining were used to evaluate TMPRSS3 and TNFRSF11B expression levels in 86 breast cancer samples. Tissue samples stained for TMPRSS3 and TNFRSF11B expression were classified into five categories and given a score from 0 to 5 according to the percentage of positively stained cells in each sample: '0' (0\%), ' 1 ' (1-25\%), '2' (>25-50\%), '3' (>50-75\%) and '4' (>75-100\%). Additionally, the staining intensity of tissue samples was used to divide them into four categories and assign them a score between 0 and 3: 0 , negative; 1 , weak; 2 , moderate; and 3 , strong. Then, the product of the first and second score was used to determine TMPRSS3 and TNFRSF11B expression levels.

Statistical analysis. The results are expressed as mean \pm SD (standard deviation). Statistical significance between the groups was assessed using one-way analysis of variance (ANOVA). Univariate survival and multivariate analyses were carried out using the Kaplan-Meier method. All calculations were performed using the SPSS 20.0 software program (SPSS, Inc., Chicago, IL, USA). The level of significance was chosen as $\mathrm{P}<0.05$.

\section{Results}

Gene microarray analysis. We downloaded a 286 breast cancer gene-expression profile dataset of Wang et al (16) from the GEO database (GSE2034), among which 180 cases were lymph node-negative relapse-free patients and 106 were lymph node-negative patients that developed distant metastasis. Wang et al mainly discussed genes associated with distant metastasis, but we aimed to study prognosis-associated genes based on this dataset.

During the 5-year follow-up of these patients, 168 were alive, 95 were deceased and 23 were lost to follow-up. Compared to the surviving patient group, 89 genes differentially expressed in the deceased patient group displayed a 2-fold change in expression at the $\mathrm{P}<0.01$ level with a false discovery rate, among which 59 were upregulated while 30 were downregulated. CCNE2 and EEF1A2 were included in the 76-gene signature of this study to predict tumor metastasis while another 87 genes were newly discovered differential genes associated with prognosis. Then, hierarchical clustering analysis was used to detect the expression profile of 89 differentially expressed genes by MEV4.9.0 software (Fig. 1A). Kaplan-Meier survival analysis of the 89 genes showed that 46 genes displayed a log-rank $\mathrm{P}<0.05$ level in expression, among which 29 genes were upregulated while 17 genes were downregulated. The 46 genes underwent cluster analysis with MEV4.9.0 software (Fig. 1B).

We also downloaded a 260 breast cancer gene-expression profile dataset of van de Vijver et al (17) which included clinical prognostic data, for which 195 patients were alive while 65 were deceased according to the 5-year follow-up. Compared to the surviving patient group, 540 genes of the deceased patient group displayed a 2 -fold change in expression at the $\mathrm{P}<0.01$ level, among which 274 were upregulated and 266 were downregulated. Then, the 540 genes underwent cluster analysis 

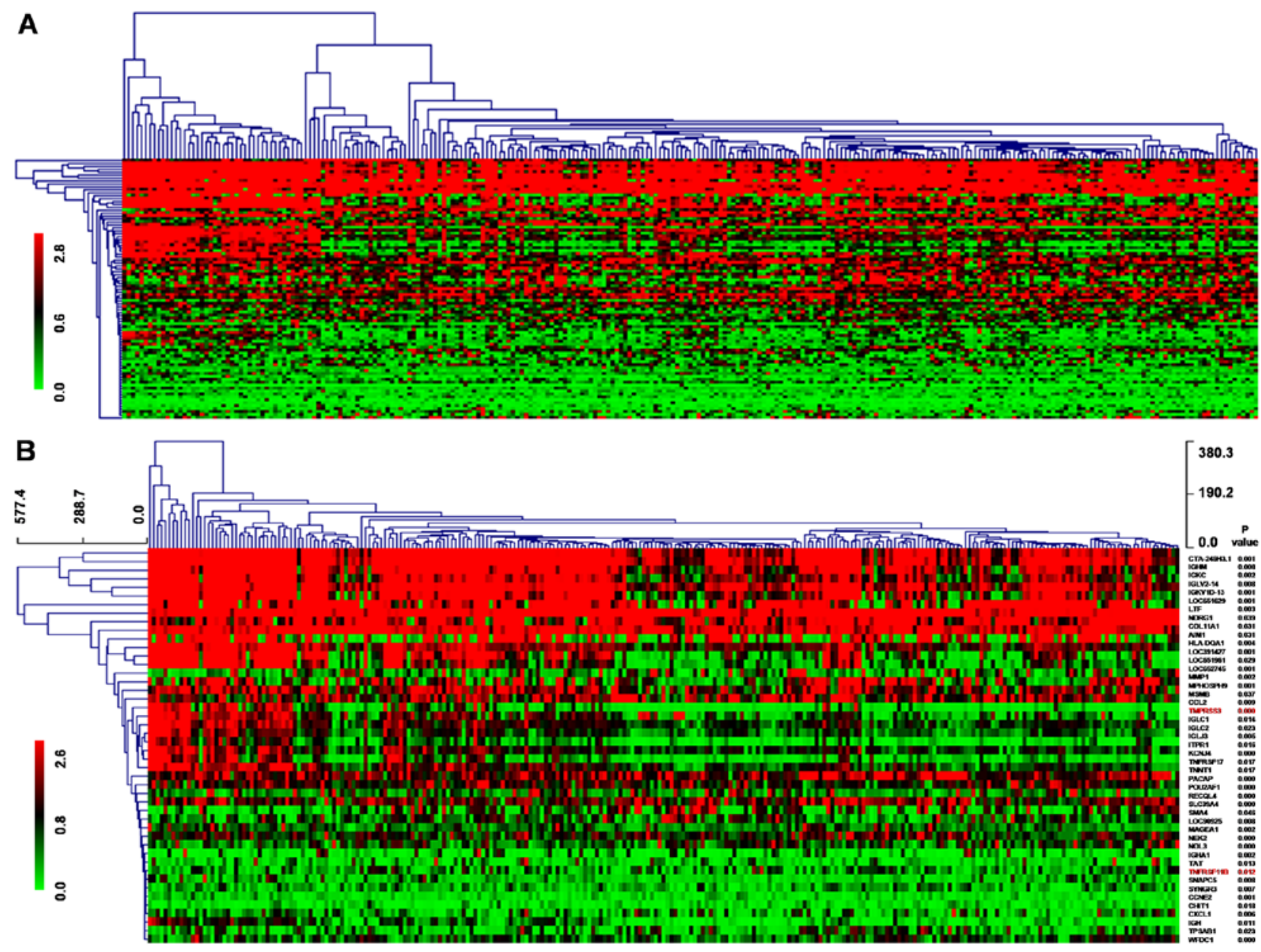

Figure 1. Hierarchical clustering of genes correlated with the prognosis of 286 breast cancer patients. (A) Hierarchical clustering of the 89 differentially expressed genes which displayed a 2-fold change in expression at the $\mathrm{P}<0.01$ level in the deceased patient group compared with the survival group. (B) Hierarchical clustering of 46 genes which displayed a log-rank $\mathrm{P}<0.05$ level in expression in the Kaplan-Meier survival analysis.

with MEV4.9.0 software (Fig. 2A). Kaplan-Meier survival analysis of the 89 genes showed that 67 genes displayed a logrank $\mathrm{P}<0.05$ level in expression, among which 45 genes were upregulated while 22 genes were downregulated. The 67 genes underwent cluster analysis with MEV4.9.0 software (Fig. 2B). Moreover, TMPRSS3 and TNFRSF11B were identified as common genes among the 46 genes from Wang et al results and the 67 genes from van de Vijver et al data.

Survival analysis of TMPRSS3 and TNFRSF11B in breast cancer. Subsequently, Kaplan-Meier survival curves were plotted to estimate the prognostic value of TMPRSS3 and TNFRSF11B in breast cancer. Firstly, we recorded TMPRSS3 and TNFRSF11B gene expression levels as high vs. low using a cut-off point according to the median. Univariate analysis of OS by Kaplan-Meier survival analysis indicated that high TMPRSS3 expression was associated with shorter OS both in the 286 breast cancer patients in Wang et al group $(\mathrm{P}<0.001$; Fig. 3A) and 260 breast cancer patients in van de Vijver et al group ( $\mathrm{P}=0.006$; Fig. 3A). However, high TNFRSF11B expression was associated with longer OS both in the 286 breast cancer patients in Wang et al group $(\mathrm{P}=0.012$; Fig. 3B) and 260 breast cancer patients in van de Vijver et al group $(\mathrm{P}=0.018$; Fig. 3B). However, these two genes were not in the list of the 93 protein-coding cancer genes which carry probable driver mutations (18). We inferred that their dysfunction may not arise by gene mutation, but by change of gene expression level.

Since TMPRSS3 and TNFRSF11B were associated with OS of the breast cancer patients, we further aimed to ascertain whether they were associated with tumor metastasis or tumor occurrence. Thus, we downloaded gene microarray dataset of Minn et al (19) including clinical information of breast cancer metastasis to lung. Distant metastasis-free survival by Kaplan-Meier survival analysis demonstrated that both TMPRSS3 and TNFRSF11B were positively correlated with breast cancer metastasis ( $\log$-rank $\mathrm{P}=0.003$; $\log$-rank $\mathrm{P}=0.024$; Fig. 3C). Lung metastasis-free survival by Kaplan-Meier survival analysis indicated that TMPRSS3 was positively correlated with breast cancer metastasis to the lung (log-rank $\mathrm{P}=0.015$; Fig. 3D), but there was no evidence to demonstrate that TNFRSF11B was correlated with breast cancer metastasis to the lung (log-rank $\mathrm{P}=0.079$; Fig. 3D). However, a previous study showed that TNFRSF11B was inversely correlated with breast cancer metastasis to the bone as it can inhibit osteoclast activity and bone metastasis (20).

Oncomine on line analysis. To further elucidate the TMPRSS3 and TNFRSF11B expression signature in breast cancer, we analyzed their expression levels in 593 breast cancer patients in the TCGA database by Oncomine. The results showed that 

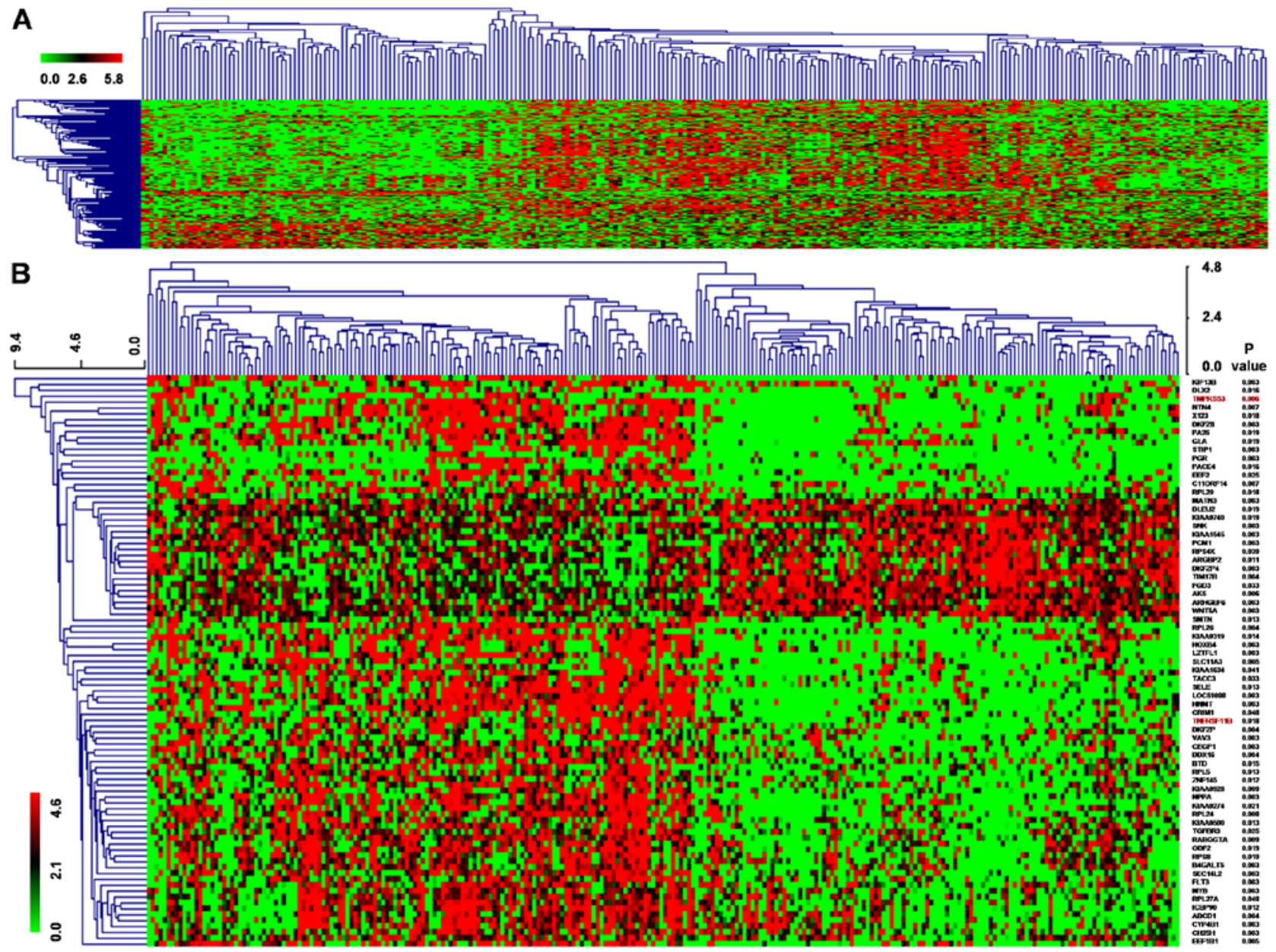

Figure 2. Hierarchical clustering of genes correlated with the prognosis of 260 breast cancer patients. (A) Hierarchical clustering of the 540 differentially expressed genes which displayed a 2 -fold change in expression at the $\mathrm{P}<0.01$ level in the deceased patient group compared with the survival group. (B) Hierarchical clustering of 67 genes that displayed a log-rank $\mathrm{P}<0.05$ level in expression in the Kaplan-Meier survival analysis.

A

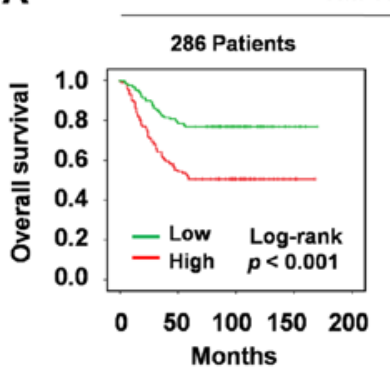

C

TMPRSS3

286 Patients

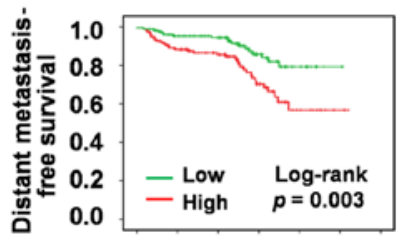

O 50100150200250300 Months
TMPRSS3

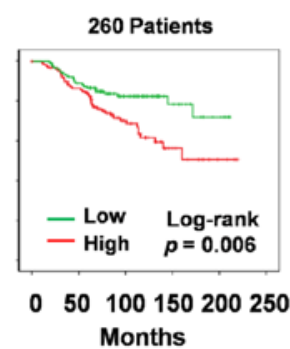

TNFRSF11B 260 Patients

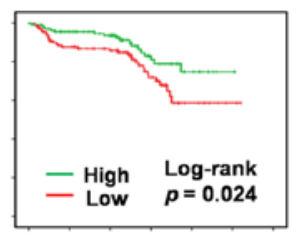

O 50100150200250300 Months
B

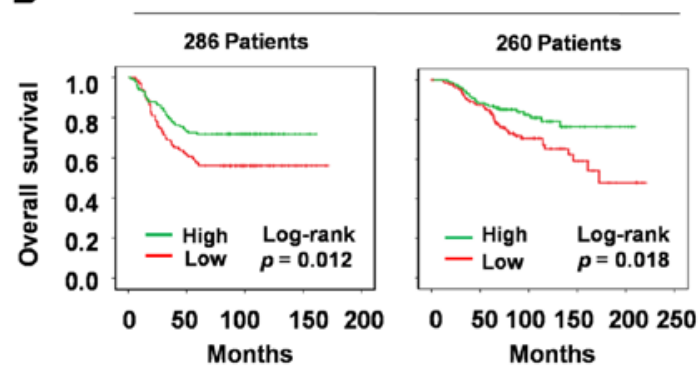

D

\begin{tabular}{c} 
TMPRSS3 \\
\hline 286 Patients
\end{tabular}

TNFRSF11B

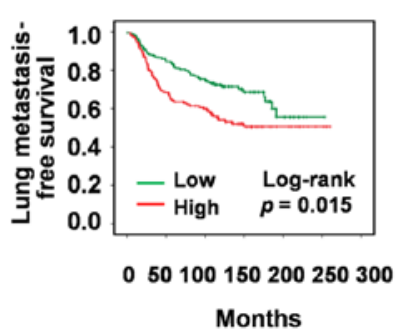

260 Patients

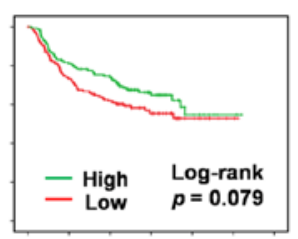

O 50100150200250300

Months

Figure 3. Survival analysis of TMPRSS3 and TNFRSF11B. (A) Univariate analysis of overall surivival (OS) by Kaplan-Meier survival analysis in breast cancer patients based on TMPRSS3 expression. (B) Univariate analysis of OS by Kaplan-Meier survival analysis in breast cancer patients based on TNFRSF11B expression. (C) Distant metastasis-free survival analysis by Kaplan-Meier survival analysis based on both TMPRSS3 and TNFRSF11B. (D) Lung metastasisfree survival analysis by Kaplan-Meier survival analysis based on both TMPRSS3 and TNFRSF11B. 
A
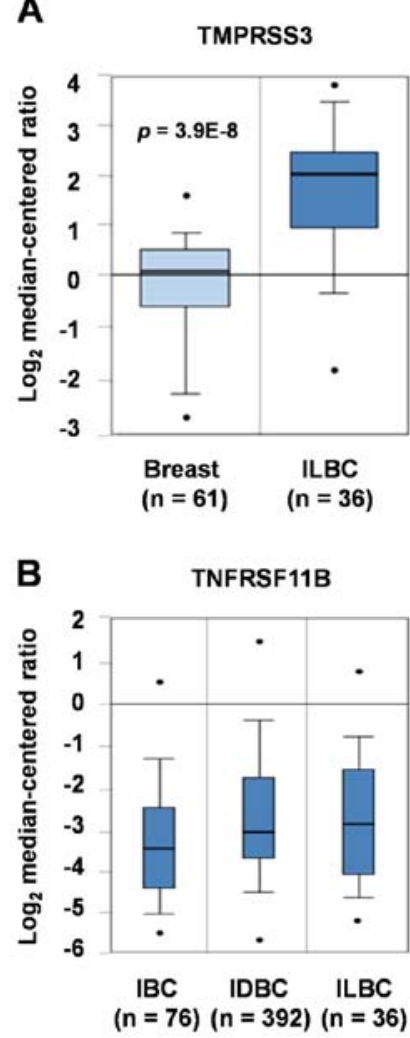

C

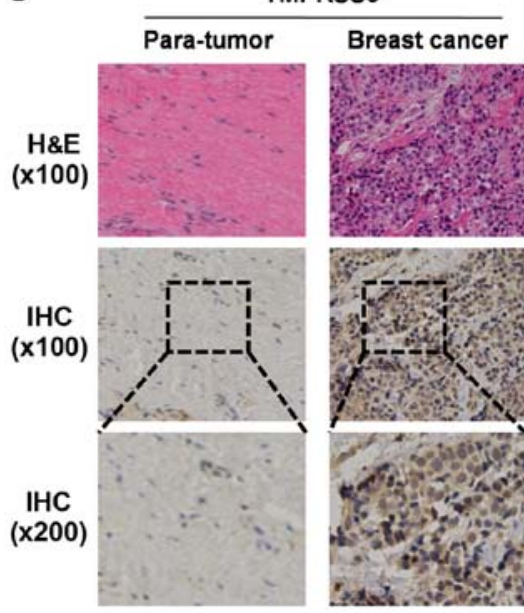

D

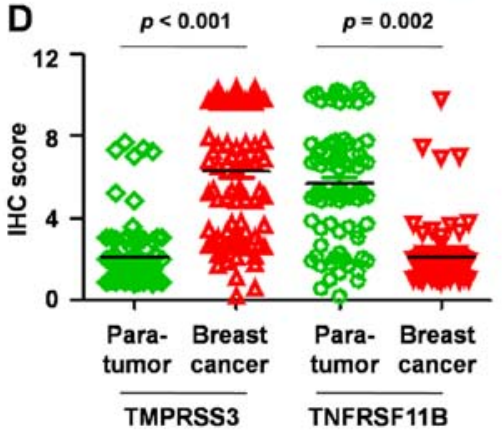

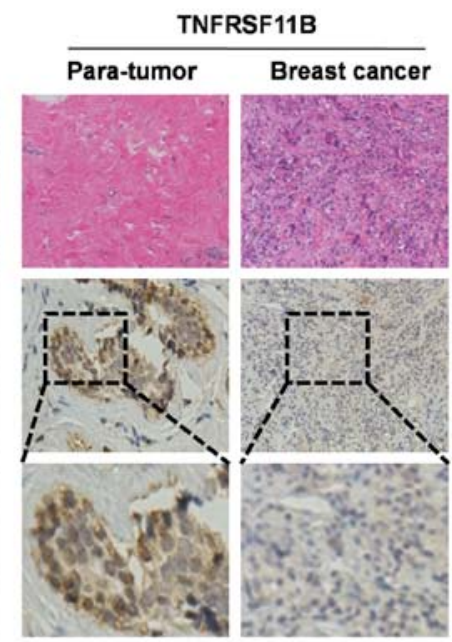

E

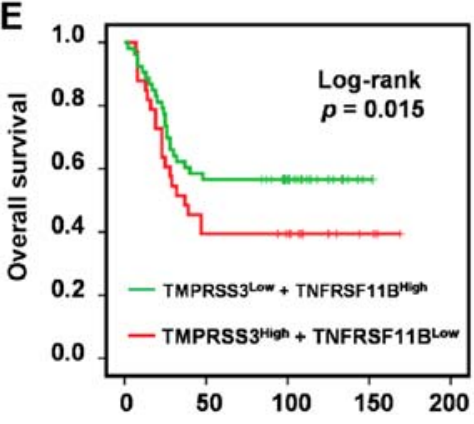

Figure 4. TMPRSS3 and TNFRSF11B expression and prognostic value. (A) Analysis of TMPRSS3 and TNFRSF11B expression levels in invasive lobular breast carcinoma (ILBC) compared with normal breast tissues in 593 breast cancer patients from the TCGA database by Oncomine. (B) Analysis of TMPRSS3 and TNFRSF11B expression levels in invasive breast carcinoma (IBC, $n=76$ ), invasive ductal breast carcinoma (IDBC, $n=392$ ), and invasive lobular breast carcinoma (ILBC; $\mathrm{n}=36$ ) in 593 breast cancer patients from the TCGA database by Oncomine. (C) Immunohistochemical (IHC) and H\&E staining of TMPRSS3 and TNFRSF11B in breast cancer. (D) IHC score of TMPRSS3 and TNFRSF11B in breast cancer vs. para-tumor tissues. (E) Multivariate analysis of overall survival (OS) with a Cox proportional hazards model in breast cancer based on TMPRSS3 and TNFRSF11B.

TMPRSS3 was significantly upregulated in invasive lobular breast carcinoma compared with that in normal breast tissue ( $\mathrm{P}=3.9 \mathrm{E}-8$; fold-change, 3.8; Fig. 4A) and TNFRSF11B was significantly downregulated in different types of breast cancer, such as invasive breast carcinoma (IBC; $n=76$ ), invasive ductal breast carcinoma (IDBC; $n=392$ ) and invasive lobular breast carcinoma (ILBC; n=36) (Fig. 4B).

$H \& E$ and IHC analysis. Moreover, H\&E staining and immunohistochemistry technique were used to analyze TMPRSS3 and TNFRSF11B expression in 86 breast cancer tissues vs. para-tumor tissues (Fig. 4C). When compared with the normal breast tissues, TMPRSS3 expression was significantly upregulated (fold-change, 3.6; $\mathrm{P}<0.001$; Fig. 4D) and TNFRSF11B expression was significantly downregulated (fold-change, 2.4; $\mathrm{P}=0.002$; Fig. 4D) in the breast cancer tissues. Next, we analyzed the correlation between TMPRSS3 and TNFRSF11B and found that the relative expression levels were inversely correlated $\left(\mathrm{R}^{2}=0.87\right.$; Fig. $\left.4 \mathrm{D}\right)$.

Clinical significance of TMPRSS3 and TNFRSF11B in 86 paired breast cancer tissues. Univariate analysis of OS showed that upregulation of TMPRSS3 expression was associated with shorter OS $(\mathrm{P}=0.033)$ while downregulation of TNFRSF11B expression was associated with shorter OS $(\mathrm{P}=0.042)$. Multivariate analysis of OS with a Cox proportional hazards model in breast cancer based on TMPRSS3 and TNFRSF11B expression demonstrated that upregulation of TMPRSS3 accompanied by downregulation of TNFRSF11B was associated with a shorter median OS and is an indicator for poor prognosis $(\mathrm{P}=0.015$; Fig. 4E).

\section{Discussion}

Breast cancer is the most commonly diagnosed cancer in women, and bone is one of the most common metastatic sites, with $\sim 75 \%$ of all women with breast cancer diagnosed with bone metastases upon autopsy examination $(21,22)$. Receptor activator of nuclear factor- $\kappa \mathrm{B}$ and its ligand (RANK/RANKL) play an important role in the development and maintenance of osteoclastic activity in bone. TNFRSF11B or osteoprotegerin (OPG) is a decoy of RANKL which blocks RANKL-RANK interaction by binding to RANKL. In this way, it acts as a natural modulator of osteoclastic activity. The RANK/ RANKL/OPG pathway plays an important role in the development and progression of bone metastasis in various types of cancers. However, little is known concerning the role of OPG in breast cancer prognosis.

Type II transmembrane serine protease (TTSP) family consisting of 17 members is a class of membrane-bound proteolytic enzymes, whose activities on the surface of tumor cells can break down surrounding extracellular matrix 
components, thereby promoting metastasis. In this way, they contribute to tumor growth, invasion and metastasis. A range of proteolytic activities occurs in malignant cells with TTSPs Thus, TTSPs are suitable candidates as tumor markers (23). TMPRSS3 is a member of the TTSP family which has been found to be highly expressed in pancreatic cancer and ovarian tumors. TMPRSS3 promotes the proliferation, invasion and migration of ovarian cancer cells $(10,11)$, and TMPRSS3 expression was found to be significantly associated with breast cancer (24). Although, it has been reported that TMPRSS3 expression is associated with the outcome of breast cancer patients, the pattern of TMPRSS3 expression in human breast cancer remains controversial.

In the present study, survival analysis of OS in two groups of gene-expression profile datasets showed that TMPRSS and TNFRSF11B were common prognostic-associated genes in breast cancer. Further univariate analysis of OS, distant metastasis-free and lung metastasis-free survival based on TMPRSS3 and TNFRSF11B expression in breast cancer demonstrated that high TMPRSS3 expression and low TNFRSF11B expression were associated with shorter OS, and they were positively correlated with breast cancer metastasis. While TMPRSS3 was positively correlated with breast cancer metastasis to the lung, there was no evidence to demonstrate that TNFRSF11B was correlated with breast cancer metastasis to the lung. Next, we explored their expression levels in breast cancer vs. normal breast tissues in the TGCA database using Oncomine and verified the results with $\mathrm{H} \& \mathrm{E}$ and IHC techniques. The results showed that TMPRSS3 was upregulated and TNFRSF11B was downregulated in breast cancer. Moreover, TMPRSS3 and TNFRSF11B expression levels presented an inverse correlation.

Finally, multivariate analysis of OS based on TMPRSS3 and TNFRSF11B expression demonstrated that upregulated TMPRSS3 accompanied with downregulated TNFRSF11B was associated with a shorter median OS and was indicative of a poor prognosis. Therefore, high TMPRSS3 and low TNFRSF11B expressions are independent prognostic factors for poor breast cancer survival. However, the molecular mechanisms of TMPRSS3 and TNFRSF11B in breast cancer and bone metastasis remain unclear.

Taken together, TMPRSS3 and TNFRSF11B are independent prognostic factors that may be used as potential biomarkers to identify subgroups of breast cancer tumors with different degrees of aggressiveness. They could also serve as prognostic markers and even potential therapeutic targets for breast cancer patients in the future.

\section{Acknowledgements}

The present study was supported partly by grants from the National Natural Science Foundation of China (nos. 81201535, 81472202 and 81302065), the Shanghai Natural Science Foundation (12ZR1436000 and 16ZR1428900) and the Shanghai Municipal Commission of Health and Family Planning (201440398 and 201540228).

\section{References}

1. Nattinger $A B$ and Mitchell JL: Breast cancer screening and prevention. Ann Intern Med 164: ITC81-ITC96, 2016.
2. Siegel RL, Miller KD and Jemal A: Cancer statistics, 2016. CA Cancer J Clin 66: 7-30, 2016.

3. Fan L, Strasser-Weippl K, Li JJ, St Louis J, Finkelstein DM, Yu KD, Chen WQ, Shao ZM and Goss PE: Breast cancer in China. Lancet Oncol 15: e279-e289, 2014.

4. Prat A, Cheang MC, Galván P, Nuciforo P, Paré L, Adamo B, Muñoz M, Viladot M, Press MF, Gagnon R, et al: Prognostic value of intrinsic subtypes in hormone receptor-positive metastatic breast cancer treated with letrozole with or without lapatinib. JAMA Oncol 2: 1287-1294, 2016.

5. Maciejczyk A: New prognostic factors in breast cancer. Adv Clin Exp Med 22: 5-15, 2013.

6. Recondo G Jr, de la Vega M, Galanternik F, Díaz-Cantón E, Leone BA and Leone JP: Novel approaches to target HER2positive breast cancer: Trastuzumab emtansine. Cancer Manag Res 8: 57-65, 2016.

7. Bianco $S$ and Gévry N: Endocrine resistance in breast cancer: From cellular signaling pathways to epigenetic mechanisms. Transcription 3: 165-170, 2012.

8. Giessrigl B, Schmidt WM, Kalipciyan M, Jeitler M, Bilban M, Gollinger M, Krieger S, Jäger W, Mader RM and Krupitza G: Fulvestrant induces resistance by modulating GPER and CDK6 expression: Implication of methyltransferases, deacetylases and the hSWI/SNF chromatin remodelling complex. Br J Cancer 109: 2751-2762, 2013.

9. Marquette C and Nabell L: Chemotherapy-resistant metastatic breast cancer. Curr Treat Options Oncol 13: 263-275, 2012.

10. Wallrapp C, Hähnel S, Müller-Pillasch F, Burghardt B, Iwamura T, Ruthenbürger M, Lerch MM, Adler G and Gress TM: A novel transmembrane serine protease (TMPRSS3) overexpressed in pancreatic cancer. Cancer Res 60: 2602-2606, 2000.

11. Zhang D, Qiu S, Wang Q and Zheng J: TMPRSS3 modulates ovarian cancer cell proliferation, invasion and metastasis. Oncol Rep 35: 81-88, 2016.

12. Rui X, Li Y, Jin F and Li F: TMPRSS3 is a novel poor prognostic factor for breast cancer. Int J Clin Exp Pathol 8: 5435-5442, 2015.

13. Pelkonen M, Luostari K, Tengström M, Ahonen H, Berdel B, Kataja V, Soini Y, Kosma VM and Mannermaa A: Low expression levels of hepsin and TMPRSS3 are associated with poor breast cancer survival. BMC Cancer 15: 431, 2015.

14. Lacey DL, Timms E, Tan HL, Kelley MJ, Dunstan CR, Burgess T, Elliott R, Colombero A, Elliott G, Scully S, et al: Osteoprotegerin ligand is a cytokine that regulates osteoclast differentiation and activation. Cell 93: 165-176, 1998.

15. Dougall WC and Chaisson M: The RANK/RANKL/OPG triad in cancer-induced bone diseases. Cancer Metastasis Rev 25: 541-549, 2006.

16. Wang Y, Klijn JG, Zhang Y, Sieuwerts AM, Look MP, Yang F, Talantov D, Timmermans M, Meijer-van Gelder ME and Yu J: Gene-expression profiles to predict distant metastasis of lymph-node-negative primary breast cancer. Lancet 365: 671-679, 2005.

17. van de Vijver MJ, He YD, van't Veer LJ, Dai H, Hart AA, Voskuil DW, Schreiber GJ, Peterse JL, Roberts C, Marton MJ, et al: A gene-expression signature as a predictor of survival in breast cancer. N Engl J Med 347: 1999-2009, 2002.

18. Nik-Zainal S, Davies H, Staaf J, Ramakrishna M, Glodzik D, Zou X, Martincorena I, Alexandrov LB, Martin S, Wedge DC, et al: Landscape of somatic mutations in 560 breast cancer whole-genome sequences. Nature 534: 47-54, 2016.

19. Minn AJ, Gupta GP, Siegel PM, Bos PD, Shu W, Giri DD, Viale A, Olshen AB, Gerald WL and Massagué J: Genes that mediate breast cancer metastasis to lung. Nature 436: 518-524, 2005.

20. Park HR, Min SK, Cho HD, Kim DH, Shin HS and Park YE: Expression of osteoprotegerin and RANK ligand in breast cancer bone metastasis. J Korean Med Sci 18: 541-546, 2003.

21. Suva LJ, Washam C, Nicholas RW and Griffin RJ: Bone metastasis: Mechanisms and therapeutic opportunities. Nat Rev Endocrinol 7: 208-218, 2011.

22. Chen X, Lu J, Ji Y, Hong A and Xie Q: Cytokines in osteoblast-conditioned medium promote the migration of breast cancer cells. Tumour Biol 35: 791-798, 2014.

23. Hooper JD, Clements JA, Quigley JP and Antalis TM: Type II transmembrane serine proteases. Insights into an emerging class of cell surface proteolytic enzymes. J Biol Chem 276: 857-860, 2001.

24. Luostari K, Hartikainen JM, Tengström M, Palvimo JJ, Kataja V, Mannermaa A and Kosma VM: Type II transmembrane serine protease gene variants associate with breast cancer. PLoS One 9: e102519, 2014. 